\title{
Führt die funktionelle Beanspruchung der Lungen beim Spielen von Blasinstrumenten zu Emphysem?
}

Von

Erich Becker.

Mit 1 Kurve im Text.

Die für die Ätiologie des Emphysems in Betracht kommenden Theorien kann man zweckmässig - einer hergebrachten Einteilung zufolge - in zwei grosse Gruppen scheiden; einmal werden pathologisch-anatomisch nachweisbare Veränderungen der Lungen selbst als ursächliche Momente genannt; auf der anderen Seite dagegen misst man der mechanischen Entstehung - also der Lungendehnung - durch Erschwerung der Ein- oder Ausatmung eine wesentliche Bedeutung zu. Mit der letzteren Ersache des Emphysems durch Erschwerung der Ausatmung beschäftigen wir uns in folgendem.

Der Frage, ob durch die Ausübung verschiedener Berufsarten, wie z. B. Glasblasen oder durch Spielen von Blasinstrumenten ein echtes Emphysem entstehen könne, ist man verschiedentlich näher getreten. Es sei mir gestattet, die hier in Betracht kommenden Arbeiten und ihre Ergebnisse kurz anzuführen.

Pretin und Leibkind untersuchten im ganzen 230 Glasbläser auf die Frage hin, ob tatsächlich in diesem Berufe Emphysem sich so häufig fand, wie man - nach allgemein gültigen Anschauungen - bisher anzunehmen geneigt war. Bläser, die weniger als 10 Jahre im Berufe tätig waren, wurden von vornherein von der Untersuchung ausgeschaltet. Die Mehrzahl der Bläser waren zwischen 11 und 20 Jahren im Dienst.

Die Methode dieser beiden Autoren bestand in „Messung des Thoraxumfangs mit dem Bandmass, durch Perkussion wurde Stand 
und Verschieblichkeit des Zwerchfells bestimmt und der auskultatorische Befund der Lungen erhoben. Spirometrisch wurde die Vitalkapazität gemessen und deren Wert im Verein mit den übrigen Befunden bei der Entscheidung ob Emphysem vorlag, in Betracht gezogen. Die gefundenen Spirometerwerte werden im Mittel zu $3300 \mathrm{~cm}$ angegeben."

Die Ergebnisse dieser Untersuchungen sind in kurzen Zügen folgende: unter 164 Bläsern konnte kein Fall von Emphysem ermittelt werden; bei 54 Bläsern im Alter von 40-50 Jahren konnte nur in 2 Fällen „ein nicht einmal deutlich ausgeprägtes Emphysem" konstatiert werden. Von den 12 ältesten Bläsern boten drei das klinische Bild des Emphysems. „Im ganzen also unter 230 Fällen nur fünf wirkliche Emphysematöse"! Daraus ziehen Pretin und Leibkind den Schluss, ,dass der Beruf als Glasbläser kein ätiologisches Moment für die Entstehung des Emphysems abgibt." „Eher zeigen bei den Glasbläsern die Lungen entschieden eine Angewöhnung an die Anstrengung des Blasens." Ein weit wichtigeres, die Entstehung des Emphysems begünstigendes Moment sehen Pretin und Leibkind darin, dass mit dem Beruf ein ständiger Aufenthalt in schlecht gelüfteten, mit Staub und Rauch erfüllten Räumen, der zu hartnäckiger katarrhalischer Bronchitis Veranlassung gibt, verbunden ist. Die Erweiterung der Lungenalveolen durch die Erschwerung der Exspiration genügt bei gesunder Lunge nicht, ein Emphysem zu erzeugen, es müssen stets organische Veränderungen des Lungengewebes hinzukommen; nur dem Zusammenwirken beider Faktoren kanm die Erzeugung eines echten Emphysems zur Last gelegt werden.

$\mathrm{Zu}$ genau dem gleichen Ergebnis kommt H. Fischer durch die klinische Untersuchung ron 500 Vilitärmusikern. Auch die älteren ron ihm untersuchten Bläser ,zeigten keineswegs den Symptomenkomplex des Lungenemphysems". Das ausgebildete Emphysem konnte er selbst bei den am längsten im Dienst und Beruf befindlichen Musikern nirgends mit Sicherheit konstatieren. Auch F is c her neigt der Ansicht zu, dass mit dem Spielen von Blasinstrumenten eine Übung der Lungen verbunden ist. Der Aufenthalt in rauchigen, staubigen Lokalen, die meist dabei in erheblichen Quantitäten vertilgten alkoholischen Getränke und die durch dies Leben verursachto chronische Bronchitis sind eher als ursächliche Momente zu beschuldigen, als das Spielen von Blasinstrumenten.

Die Yöglichkeit, dass durch die Ausübung verschiedener Berufo ein substantielles Emphysem erzeugt werden könne, gibt auch F. A. Hoffmann zu, wenn er in seiner Monographie „Emphysem 
und Atelektase" sagt: ,unter den Beschäftigungsarten sind diejenigen zu fürchten, welche erhebliche und oft wiederholte Anstrengungen mit Steigerung von Druck auf die Lunge zur Folge haben. So gilt vor allen Dingen das Spielen von Blasinstrumenten als schädlich, hier wird die freie Zirkulation der Luft durch das vor den Mund genommene Hindernis geschädigt. Aber es ist doch darauf hinzuweisen, dass ein vernünftiges Blasen sehr wohl so geübt werden kann, dass die Lungen dabei nicht geschädigt werden und wir haben in allen Kapellen ältere, sehr tüchtige und leistungsfähige Bläser. Allerdings müssen sie sehr darauf achten, ihrer Lunge nichts über das Mass zuzumuten und namentlich nach schwächenden Krankheiten nicht zu bald wieder mit der gewohnten Energie zu blasen. Leicht verständlich ist daher der Fall von jenem Trompeter, der nach einer Pneumonie ein Emphysem der ganzen früher pneumonisch infiltrierten Lunge bekam."

„In ähnlicher Weise ist das Glas- und Lötrohrblasen zu beurteilen, auch hier finden wir, dass der geschickte und geübte Bläser, wenn er sonst gesund ist, ungestraft seiner Tätigkeit obliegen kann."

Ferner bekennt sich Tendeloo in seinem klassischen Werke .Über die Ursachen der Lungenkrankheiten“ zu der Ansicht, dass die Ausübung verschiedener Berufe ätiologische Momente für die Entstehung des Lungenemphysems abgebe. „Es fusst der mechanische Erklärungsversuch des Emphysems, welcher seine Ursache in mechanischen oder dynamischen Störungen der Atmung erblickt, auf einigen unverkennbaren Tatsachen". Unter den Berufen, die mit Störungen im Mechanismus der Atmung einhergehen, nennt Tendel o o Blasmusiker, Glasbläser, Marktschreier, Sänger, Prediger usw.

Der pathologisch-anatomische Prozess bestehe in einer Überdehnung der elastischen Lungenєlemente, die sich durch öftere Wiederholung der akuten Lungenblähung entwickle.

Erwähnt sei noch, dass auch Tendeloo der Ansicht ist, dass bei Entstehung des Emphysems neben individueller Disposition noch andere Faktoren eine ausschlaggebende Rolle spielen, so sei z. B. der Widerstand des Mundstückes bei den verschiedenen Instrumenten verschieden, ferner verstehe es der eine Bläser besser seinen Atem zu „verteilen“ als der andere, und endlich sind Ausbildung und physikalische Eigenschaften des elastischen Gewebes in den Lungen grossen individuellen Schwankungen unterworfen.

Das durch Exspirationsstenose erzeugte Emphysem macht sich nach Tendeloo zunächst, ,in den suprathorakalen Teilen bemerkbar. Der oftmaligen akuten Blähung folgt ein Zustand dauernder 
Überdehnung und Vergrösserung. Natürlich erweitert sich auch der Thorax bei der akuten Lungenblähung. Diese zunächst kurz anhaltende Formreränderung des Thorax wird aber bei häufiger Wiederholung der Dehnung dauernd; aus dem rordem normalen Thorax bildet sich ein fassförmiger."

Bei der klinischen Untersuchung der ron uns untersuchten Musiker rurde auf die Vorwölbung und das Verstrichensein der Supra- und Infraklarikulargruben, sowie auf Starrheit des Thorax im ganzen geachtet, es liess sich aber ein derartiger Befund in keinem der von uns untersuchten Fälle nachweisen.

In den Verhandlungen des Kongresses für innere Medizin berichtet neuerdings Lom mel unter dem Titel, .Zur Pathogenese des Emphysems" über eine Untersuchungsreihe an den Glasbläsern Jenas.

Lommel benutzte, was sämtliche bisher genannte Autoren nicht taten, als weitere, die klinische Untersuchung ergänzende Methode die Spirometrie. Die einzelnen Komponenten des Lungenluftwechsels wurden nach $\mathrm{B}$ oh $\mathrm{r}$ bestimmt, die Residualluft nach der Wasserstoffmischmethode Darys. Neben der spirometrischen wurde die klinische Untersuchung geübt, ausserdem die Zwerchfellbewegung orthodiagraphisch festgestellt.

Dic Ergebnisse der spirometrischen Versuche Lommels stimmten - was die absoluten erhaltenen Werte anlangt - mit denen anderer Autoren überein; wichtiger als diese absoluten Werte für einzelne Atemvolumina erscheinen ihm die Relationen zwischen den Volumenergebnissen. so besonders das Verhältnis ron Mittellage zu Totalkapazität und Residualluft zu Totalkapazität.

Das Verhältnis Mittellage zu Totalkapazität, das ron $\mathrm{B}$ ohr in der Norm zu 620\% angegeben wird, fand Lo $\mathrm{m} \mathrm{mel}$ bei seinen Untersuchungen auf $70-76^{\circ}$; erhöh. Ebenso wies auch die Relation Residualluft zu Totalkapazität grössere Werte auf. Der von Bohr verzeichnete Normalwert von $30 \%$ wurde ,teils wenig, teils bedeutend" vermehrt gefunden auf 45 und $56 \%$. Damit ist eine dauernde vermehrte Inspirationsstellung des Thorax unzweifelhaft nachgewiesen.

Als echtes chronisches Lungenemphysem möchte es Lommel nicht beztichnen. Die rermehrte Inspirationsstellung erscheint ihm aber als prädisponierender Faktor in der Pathogenese des Emphysems.

Wir sehen also, dass eine einheitliche Beurteilung der Ätiologie des Berufsemphysems in keiner Weise vorliegt. Auf Anregung von Oberarzt Dr. Bruns habe ich es unternommen, die Frage, ob durch Ausübung eines Berufes, der mit häufig wiederholter aluter Lungen- 
blähung einhergeht, Emphysem entstehen könne, durch klinische und spirometrische Untersuchung nochmals zu bearbeiten.

Die Untersuchungen wurden rorgenommen an 22 Musikern.

Die ron uns angewandte Methode der Spirometrie, auf die neben der klinischen Untersuchung das Hauptgewicht gelegt wurde, war folgende:

Wir benutzten bei unseren Versuchen ein nach den B o h $\mathrm{r}$ schen Originalangaben vom Mechaniker $\mathrm{R}$ in $\mathrm{k}$ des hiesigen physiologischen Instituts in mustergültiger Weise rerfertigtes Spirometer. Dasselbe fasst 10 Liter, hat eine sehr leicht bewegliche Glocke, die aber trotz der leichten Beweglichkeit sehr gut gegen ein zwischen zwei senkrechten Schienen sich bewegendes Laufgewicht ausgewogen sein muss. An der Seite des Spirometers ist eine Millimeterteilung angebracht, es entspricht bei unserem Apparat $1 \mathrm{~mm}$ Höhenrerschiebung: der Spircmeterglocke $25 \mathrm{ccm}$ gewechselten Luftrolumens.

Zur Bestimmung der Residualluft brachten wir die Davysche Wasserstoffmischmethode in Anwendung. Die sämtlichen Atemvolumina wurden in einem Versuche bestimmt nach der von $\mathrm{H}$ a s se 1 bal $\mathrm{ch}$ angegebenen Technik.

Lie Versuchsperson sass in einem bequemen Lehnstuhl, der Mund war in gleicher Höhe wie das mittels durchbohrten Gummistopfens an das Zuleitungsrohr des Spirometers angedichtete Mundstück. Die Nasenatmung wurde durch eine federnde, gut geglättete und abgerundete Holzklammer ausgeschaltet. Dass strengstens darauf geachtet wurde, dass die Versuchsperson mindestens 5-6 Stunden nicht geblasen hatte, damit eine vielleicht noch bestehende akute Lungenblähung keine Fehlerquellen ergebe, sei nebenbei erwähnt.

Die Bewegungen der Spirometerglocke murden auf ein $\mathrm{Lud}$. wigsches Kymographion auf berusstes Papier aufgezeichnet (cf. die beiliegende Kurve) ${ }^{1}$ ).

Der Verlauf eines Versuchs gestaltete sich folgendermassen:

Das gut durchlüftete Spirometer wird mit 3 Litern reinem; aus einer Bombe entnommenen Sauerstoff beschickt, dann auf 6 Liter mit atmosphärischer Luft aufgefüllt. Reinen Sauerstoff benutzten wir aus dem Grunde, damit die Fehlerquelle einer etwa nicht ausreichenden Verbrennung des Wasserstoffs von vornherein ausgeschaltet wurde. Den erforderlichen Wasserstoff stellten wir.aus käuflichem arsenfreiem Zink im Ki p p schen Apparat dar, leiteten zur grösseren Sicherheit den entwickelten Wasserstoff durch zwei Waschflaschen,

) Die in die Kurve eingezeichneten Zahlen geben die im Original gemessenen Ordinatenhühen an. 


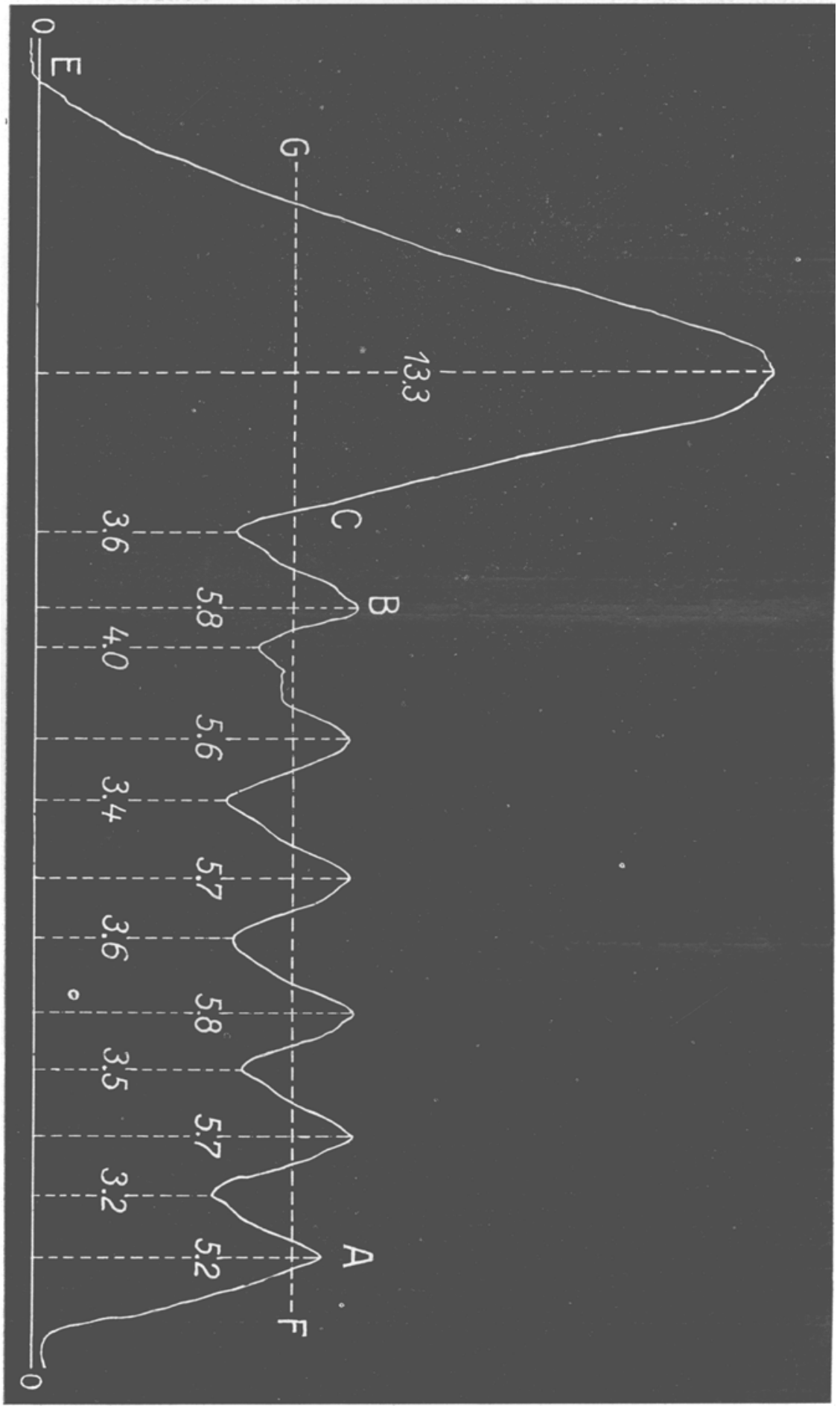


die, mit Kaliumpermanganat angefüllt, jede Spur von giftigen Arsenwasserstoffen zurückbehalten sollten. In das Spirometer liessen wir, nachdem eine Zeitlang das sich entwickelnde Gas die Luft des K i p p schen Apparats verdrängt hatte und in die freie Luft geströmt war, durch Umstellung des Spirometerdreiweghahnes ca. $1-11 / 2$ Liter Wasserstoff einströmen. Das Spirometer blieb dann 10 Minuten stehen, damit die Gase in ihm zur innigen Mischung Zeit hätten; ablürrzen lässt sich diese Zeit durch kleine stossweise ausgeführte Bewegungen der Spirometerglocke bei geschlossenem Hahn. Beror wir unsere Untersuchungen an den Musikern begannen, haben wir uns vielfach davon überzeugt, dass ein 10 Minuten langes Stehen des Spirometers verbunden mit einigen kleinen Bewegungen der Glocke genügt, um eine innige Mischung der Gase zu gewährleisten. Ausserdem machten wir oft Bestimmungen aus verschiedenen Höhen des Spirometers, mussten uns aber durch genau die gleichen Resultate der Verbrennung überzeugen, dass die Mischung der Gase eine vollkommene war.

Vor Beginn des Versuchs wurde nun der Prozentgehalt an Wasserstoff in dem Spirometer bestimmt, indem $100 \mathrm{ccm}$ Gasgemisch in eine Gasbürette geleitet wurden und von da zur Verbrennung in einen kleinen Verbrennungsapparat, in dem das Knallgasgemisch an einer mittels elektrischen Stromes glühend gemachten Platinspirale zur Explosion gebracht wurde.

Durch Zurückleiten in die Gasbürette wurde jetzt die Verminderung der Gasmenge festgestellt. Da sich aus den beiden Gasen Wasser gebildet hat, ist die Zahl der weniger abgelesenen ccm so auf die einzelnen Gase zu verrechnen, dass $2 / 3$ auf den Wasserstoff, $1 / 3$ auf Sauerstoff kommt. Durch Multiplikation des abgelesenen Wertes mit $2 / 3$ wird also sofort die Prozentzahl gefunden.

Nachdem weiterhin der Inhalt des Spirometers an der Skala abgelesen ist, ist alles zur Anstellung eines Versuches bereit.

Die Versuchsperson setzt sich bequem in dem Stuhl zurecht, Mund genau in Höhe des Spirometerhahnes, nimmt das mit Alkohol desinfizierte Mundstück zwischen die Lippen und die Zähne; die Nase wird abgeklemmt und nun atmet die Versuchsperson ruhig 10-15 Atemzüge durch den auf die Seitenöffnung eingestellten Dreiweghahn in die Aussenluft ein und aus. Auf Aufforderung des Beobachters erfolgt dann eine tiefe Einatmung, darauf eine ti $\mathrm{e}$ s $\mathrm{s}$ te Ausatmung. In der tiefsten Exspirationsstellung, die die Versuchsperson durch Heben eines Fingers markiert, muss einen Augenblick ruhig verharrt werden. Der Beobachter stellt in diesem Moment durch schnelle Drehung den Hahn auf Verbindung der Versuchs- 
person und Spirometer und Abschluss der Nebenöffnung, es besteht dann, vorausgesetzt, dass die Versuchsperson keine Luft neben dem Mundstück entweichen lässt, eine luftdichte Verbindung zwischen Spirometer und Versuchsperson. Es folgen jetzt 5-6 Atemzüga von gewöhnlicher Tiefe, dann eine tiefste In- und tiefste Exspiration, nach der das Spirometer unmittelbar ganz abgeschlossen wird. Diese tiefste Exspiration muss den Spirometerschreibstift wieder auf idie gleiche Höhe einstellen, wie sie zu Anfang des Versuches stand, die Versuchsperson kam ja auch in tiefster Exspiration an den Apparat. Als ein Kennzeichen eines gelungenen Versuchs ist es mithin zu betrachten, wenn die auf dem Kymographion aufgezeichnete Endlinie in die Anfangslinie ausmündet. Geringe Differenzen ron ca. $\check{0} 0 \mathrm{ccm}$ lassen sich dadurch ausgleichen, dass man zwischen dem Inhalt vor und nach dem Versuch das Mittel nimmt:

Es wird nunmehr durch Entnahme von $100 \mathrm{ccm}$ Gasgemisch und Verbrennung zu Wasser der Prozentgehalt an Wasserstoff nach dem Versuche festgestellt, in der gleichen Weise wie früher.

Der Wert des Spirometerinhaltes vor und nach dem Versuch, event. das Mittel zwischen beiden und der Prozentgehalt an Wasserstoff vor und nach dem Tersuch sind genügende Angaben zur Bestimmung der Residualluft.

Dieselbe berechneten wir aus folgender Formel:

$$
\mathrm{R}=\frac{\mathrm{J} \mathrm{h}_{1}}{\mathrm{~h}_{2}}-\mathrm{J}
$$

worin $\mathrm{R}$ den Wert der Residualluft, $\mathrm{h}_{1}$ den Wasserstoffgehalt vor dem Versuche in Prozenten, $h_{2}$ den Wasserstoffgehalt in Prozenten nach dem Versuch bedeutet.

$J$ ist der Inhalt des Spirometers (event. das Mittel zwischen Inhalt vor und nach dem Tersuch bei kleinen Differenzen).

Es erklärt sich diese Formel auf folgende Weise: bekannt sind der Inhalt des Spirometers und die prozentuale Wasserstoffmengra in ihm, mithin auch die absolute in dem Spirometer befindliche Wasserstoffmenge als das Produkt aus Inhalt mal Prozentgehalt an Wasserstoff. Da nun die Versuchsperson in tiefster Exspiration an den Apparat kommt, mit anderen Worten nur mit der Residualluft in den Lungen, so muss - gleich tiefste Exspiration rorausgesetzt der absolute Inhalt des Systems Spirometer und Lungen der Versuchsperson vor und nach dem Tersuche gleich sein. Ein Teil des in dem Spirometer befindlichen Wasserstoffs wird zur Mischung mit der Residualluft verbraucht, das Mischungsverhältnis der Lungenluft mit Wasserstoff muss nach einer genügenden Anzahl von Atem- 
zügen aus dem Spirometer in den Lungen das gleiche sein, wie im Spirometer. Bezeichnen wir die prozentuale Wasserstoffmenge am Ende des Versuchs mit $h_{2}$, so enthält die in den Lungen befindliche Residualluft $\mathrm{R}$ am Ende des Versuchs $\mathrm{Rh}_{2}$ Wasserstoff in absolut gemessener Menge, das Spirometer in gleicher Weise $\mathrm{J} \mathrm{h}_{2}$.

Die Summe dieser beiden Wasserstoffmengen muss gleich sein der absoluten Wasserstoffmenge des Systems Spirometer und Iungen der Versuchsperson vor dem Versuch, ein Verlust an Wasserstoff ist ja in diesem System nicht eingetreten, nur das Mischungsverhältnis der gleich gebliebenen absoluten Menge $J_{h_{1}}$ hat sich geändert; es ergibt sich daraus

$$
\mathrm{R}_{2}+\mathrm{J} \mathrm{h}_{2}=\mathrm{J} \mathrm{h}_{1} \text {. }
$$

Diese Gleichung nach der einzigsten Cnbekannten $\mathrm{R}$ aufgelöst, ergibt:

$$
\begin{gathered}
\mathrm{h}_{2}(\mathrm{R}+\mathrm{J})=\mathrm{J} \mathrm{h}_{1} \\
\mathrm{R}+\mathrm{J}=\frac{\mathrm{J} \mathrm{h}}{\mathrm{h}_{2}} \\
\mathrm{R}=\frac{\mathrm{J} \mathrm{h}_{1}}{\mathrm{~h}_{2}}-\mathrm{J}
\end{gathered}
$$

Die anderen Atemvolumina sind von der auf dem berussten Papier gezeichneten Kurve zu entnehmen. Da $1 \mathrm{~mm}$ Höhenverschiebung der Spirometerglocke $25 \mathrm{ccm}$ gewechselten Luftrolumens entspricht, kann man durch Ausmessung der Kurve mit einem Anlagemassstab leicht die übrigen Volumina, wie Vitalkapazität, Reserveluft, Komplementärluft bestimmen.

Der Einfachheit halber sei an einem Beispiel die Ausrechnung: dieser Atemvolumina demonstriert (cf. Kurve). Der Wert der Reserveluft wird auf folgende Weise berechnet: Die Differenz des Mittels der höheren Ordinaten und des Mittels der kleineren Ordinaten ergibt das im Mittel mit einem der gewöhnlichen Atemzüge im Abschnitt A B der Kurve gewechselte Volumen, zunächst ausgedrückt als Höhenverschiebung der Spirometerglocke in Millimetern. Da nun $1 \mathrm{~mm}$ gleich ist $25 \mathrm{ccm}$ gewechselten Luftrolumens, so ist aus diesen Angaben leicht das Volumen eines der normalen Atemzüge zu bestimmen. Die Hälfte der Höhenverschiebung der Spirometerglocke bei diesem mittleren Atemzuge addiert zu dem Mittel der kleineren Ordinaten ergibt in Millimetern ausgedrückt, die beim Wechsel der Reserveluft erfolgte Höhenverschiebung der Spirometerglooke; die Umrechnung in Volumen ist die gleiche wie oben.

Die Vitalkapazität erhält man durch Ausmessung der den Versuch beschliessenden Zacke C G E. Die hier gemessene senkrechte 
Entfernung beträgt $133 \mathrm{~mm}$, daraus berechnet sich die Vitalkapazität zu $3325 \mathrm{ccm}$. Die Differenz der Werte der Vitalkapazität und Reserveluft liefert den Wiert der Komplementärluft. Die Mittellage ist gleich der Summe von Residual- und Reserveluft. Sie ist in der Kurve dargestellt durch die punktierte Linie FG.

Sämtliche Atemvolumina der Versuchsperson sind damit gefunden, aber unter dem im Augenblicke des Versuchs herrschenden Barometerstand und der herrschenden Temperatur. In den Lungen herrscht aber der Druck Barometerstand plus Wasserdampftension bei 370. Da uns nur das Volumen, das die Luft in der Lunge wirklich einnimmt, interessieren kann, müssen sämtliche gefundenen Werte auf den in den Lungen herrschenden Druck umgerechnet werden. Weiter kann nur daran liegen das Volumen des trocknen Gases zu kennen, nicht des Gases, wie es in den Versuchen in mit Wasserdampf gesättigten Räumen gemessen wird.

Ist ein Gasvolumen über einer Flüssigkeit gemessen, mit deren Dämpfen der Raum gesättigt ist, so steht nach dem Dalt o n schen Gesetz das trockene Gas unter dem Druck, der gleich ist der Differenz des Gesamtdruckes und der Wasserdampftension bei gleicher Temperatur.

Die hier benutzte Formel ist ausführlich abgeleitet und begründet von Gebhardt, sie lautet:

$$
\mathrm{v}=\mathrm{v} \cdot \frac{1+\alpha 37}{1+\alpha \cdot \mathrm{t}} \cdot \frac{\mathrm{b}-\mathrm{s} \cdot \mathrm{t}}{\mathrm{b}-\mathrm{s} \cdot 37^{0}}
$$

dabei bedeutet

$\nabla=$ das gesuchte Volumen.

$\mathrm{v}=$ das beim Versuch gefundene Volumen.

$a=0,00367$.

$\mathrm{t}=$ Temperatur des Wassers im Spirometer nach Celsiusgraden.

s.t Wasserdampftension bei der Temperatur $t$.

s.37 = Wasserdampftension bei $30^{\circ}(=46,7)$.

$\mathrm{b}=$ der reduzierte Barometerstand.

Sämtliche in anliegender Tabelle gefundenen Werte sind nach dieser Formel auf das Volumen bei dem in den Lungen herrschenden Druck umgerechnet.

Bevor wir zur Deutung unserer Ergebnisse übergehen, sei zunächst noch einiges zur Kritik über die zur Lösung der vorliegenden Frage angewandten Methoden vorausgeschickt.

Pretin und Leibkind und Fis cher verwandten vornehmlich die physikalischen Untersuchungsmethoden. Die ersten beiden massen zugleich spirometrisch die Vitalkapazität. Ob die erhaltenen 
Werte auf den in den Lungen herrschenden Druck reduziert wurden, darüber vermissen wir eine Angabe; zudem ist zu betonen, dass die alleinige Messung der Vitalkapazität unzureichend ist, über den geblähten Zustand der Lungen Aufschluss zu geben; beim Emphysem ist der Wert der Vitalkapazität vermindert infolge der Fixation des Thorax in rermehrter Inspirationsstellung. Damit man beurteilen kamn, ob eine Lunge gebläht ist oder nicht, muss vor allen Dingen die Residualluft bestimmt werden; ihre Bestimmung im Verein mit dem Ergebnis einer erhöhten Mittellage und Herabsetzung der Vitalkapazität sind erst zur Diagnose des Emphysems ausreichend.

Verwendet man die Spirometrie, so müssen sämtliche Atemvolumina bestimmt werden, das Hauptgewicht ist auf die Bestimmung der Residualluft und Mittellage zu legen.

Die von uns angewandte Methode der Spirometrie in der von $\mathrm{Hasselbalch}$ angegebenen Technik könnte zu einem Einwand Anlass geben. Die unter starker und ungewohnter Muskelanstrengung eingenommene tiefste Exspirationsstellung erzeugt einen physiologischen Lufthunger und könnte deshalb zu grösseren Atemzügen in erhöhter Mittellage führen.

Wir haben uns nun durch eino Reihe von Versuchen darüber zu unterrichten versucht, inwieweit tatsächlich dieser Einwand berechtigt ist.

Wir liessen einen gesunden Laboratoriumsdiener, dessen Residualluft uns aus vielfachen Versuchen bekannt war, zunächst nach der $\mathrm{H}$ asselbalchschen Methode atmen und bestimmten dabei die Reserveluft zu $1440 \mathrm{ccm}$; dann nach guter Durchlüftung des Spirometers und nach Ausruhen der Versuchsperson, das gleiche Atemvolumen nach der von $\mathrm{Bohr}$ befolgten Methode, die darin besteht, dass Residualluft und Mittellage in getrennten Versuchen festgestellt werden und zwar geht $\mathrm{B}$ oh $\mathbf{r}$ bei Bestimmung der Mittellage so vor, dass die Versuchsperson am Dreiweghahn des Spirometers mehrere Minuten in die Atmosphäre atmet, und dass dann für die Versuchsperson unmerklich die Umstellung des Hahnes auf Kommunikation der Lungen der Versuchsperson mit dem Spirometer erfolgt. Eine tiefste Exspiration geht also bei dieser Methode dem Tersuche nicht vorauf. Der Wert der Residualluft betrug nach dieser Methode $1380 \mathrm{ccm}$; die erstere Methode ergibt also tatsächlich eine etwas höhere Mittellage. Durch Wiederholung dieser Versuche stellten wir fest, dass die Werte der beiden Mittellagen um meist weniger als $100 \mathrm{ccm}$ differierten. 
Zur Klärning dieser Frage ist offenbar die Bestimmung der Reserveluft ausreichend, da die Mittellage sich zusammensetzt aus den Werten für Residualluft und Reserveluft. Die Residualluft ist aber bei den kurz hintereinander folgenden Versuchen als konstant zu betrachten.

Nun die Ergebnisse unserer Tabelle!

Bei der Überlegung, welche Resultate wir zu erwarten haben, ist anzunehmen, dass die Fähigkeit zu extremer Ausatmung, d. h. maximaler Entleerung der Luft aus der Lunge, bei den Musikem durch den Beruf ganz besonders gut entwickelt ist.

In der Tat ergeben nun meine Untersuchungen, dass von 22 Musikern 19 ein an der unteren Grenze der Norm stehendes Prozentverhältnis von Mittellage und Residualluft je zur Totalkapazität aufweisen. Diese 19 Musiker blasen im allgemeinen Instrumente, deren weite Mundstücke es ermöglichen, die vor dem Intonieren eingenommene Luft rasch und rollständig bei den ersten Tönen aus den Lungen zu entleeren.

Das Terhältnis von Mittellage zu Totalkapazität gibt Bohr beim Gesunden zu 620, , bei Sportsleuten zu 540,0 an. Die meisten der von uns untersuchten Musiker stehen - was die genannten prozentualen Verhältnisse anlangt - den Sportsleuten bei Bohr gleich. Es ist damit bewiesen, das die. Mehrzahlder Musiker mit einer geringeren Lungenfüllung den Anstrengungen des Blasens gerechtzu werden vermag, als es einem andern lungengesunden Menschen möglich wäre.

Dagegen ist bei den beiden ersten Musikern meiner Tabelle das Mundstück des Blasinstruments wesentlich enger. Der dritte Musiker bläst Posaune, die zwar kein enges Mundstück hat, aber die Bewegung grosser Luftquantitäten erfordert. Es findet namentlich bei den ersten beiden Musikern eine erhebliche Pressung des exspiratorischen Luftstromes statt.

Infolge des hohen Widerstandes in dem engen Mundstück atmet der Musiker vor dem Intonieren tief ein, um die folgende anstrengende Exspiration leichter ausführen zu können. Trotz des hohen exspiratorischen Druckes ist aber die Abgabe der Luft infolge des engen Mundstückes erschwert. Die exspiratorische Druckerhöhung bedingt daher eine intensive Aufblasung der oberen Lungenteile. Bevor noch die Lunge ihre Luft abgegeben hat, wird der Musiker durch die venöse Beschaffenheit des Blutes zu einer neuen Inspiration gezwungen. Diese Einatmung ist tiefer als die erste. Es wird also 
auch durch die folgenden Einatmungen das Lungenvolumen immer mehr vergrössert.

Da also ein Musiker, der ein Instrument mit engem Mundstück bläst, bei jedem Blasen eine mehr oder weniger ausgedehnte akute Lungenblähung bekommt, so ist es nicht verwunderlich, wenn bei ihm sich im Iaufe vieler Jahre eine Überdehnung gewisser Abschnitter der Lunge einstellt. Die akute Blähung geht anfangs prompt zurück. Die jahre- und jahrzehntelange Wiederholung derselben kann eine partielle Üebrdehnung der Lungen bewirken.

Nehmen wir an, dass, wie oben betont, die Reserveluft bei unseren Versuchen um den Wert ron rund $100 \mathrm{ccm}$ zu hoch gefunden sei und berechnen wir dann die Abänderung der Prozenttabelle für dio ersten drei Nummern unserer Tabelle, so ergeben sich folgende Werte:

\begin{tabular}{c||c|c||c}
\hline & $\begin{array}{c}\text { Mittellage } \\
\text { in Prozenten } \\
\text { der Totalkapazität }\end{array}$ & $\begin{array}{c}\text { Residualluft } \\
\text { in Prozenten } \\
\text { der Totalkapazität }\end{array}$ & $\begin{array}{c}\text { Reserveluft } \\
\text { in Prozenten } \\
\text { der Vitalkapazität }\end{array}$ \\
\hline 1 & 55,3 & 35,2 & 31,1 \\
3 & 66,3 & 34,0 & 49,0 \\
60,3 & 30,5 & $\mathbf{4 3 , 4 .}$
\end{tabular}

Bei diesen drei Musikern übersteigt der absolute Wert der Residualluft den von anderer Seite fixierten Normalwert von $2 \mathrm{~L}$. Die Prozentzalnlen der Mittelkapazität und der Residualluft beregen sich an der oberen trenze der normalen Werte, oder überschreiten dieselben um ein Geringes. Diese werden von Bohr angegeben zu: Mittellage in Prozenten der Totalkapazität

$$
\text { Min. 47,1 Max. 64,5. }
$$

Residualluft in Prozenten der Totalkapazität

$$
\text { Min. 12,9 Max. 32,3. }
$$

Unser Ergebnis bei diesen drei Musikern bewegt sich in derselben Richtung wie das Ergebnis Lommels an den Glasbläsern der Jenenser Industrie. Die jugendlichen ron ihm untersuchten Bläser wiesen Zahlen auf, die denen ron trainierten Sportsleuten gleichkamen, die älteren Leute namentlich im vierten Jahrzehnt hatten eine abnorm hohe, $70-760,0$ betragende Mittellage.

Auch die Prozentzahlen der Residualluft fand Lommel ,teils wenig, teils bedeutend über den Normalwert von $30 \%$ vermehrt". 
Damit ist der Beweis geliefert, dass bei Glasbläsern noch mehr als bei unseren Musikern der Thorax in eine gewisse inspiratorische Stellung kommt. Dass dabei die Glasbläser die höheren Werte erlangen, liegt wohl in den Unterschieden des Berufes. Der Glasbläser übt die exspiratorische Pressung unstreitig häufiger und stärker aus als der Musiker.

L o m mel hebt ausdrücklich hervor, dass seine Glasbläser frei waren von allen subjektiven Beschwerden. Das gleiche fanden wir bei den von uns untersuchten Musikern. Die Beschwerden des Emphysematikers rühren eben nicht von der Überdehnung der Lungensubstanz her, sondern im wesentlichen von der begleitenden Bronchitis, der Abnahme der Pumptätigkeit des Thorax und den aus diesem mangelnden Ventilationsvermögen sekundär sich herausbildenden Herz- und Kreislaufsanomalien.

Zum Schluss wollen wir versuchen, die Ergebnisse der Spirometrio einmal gegenüber zu halten den Ansichten Tendeloos, die derselbe auf Grund langjähriger pathologisch-anatomischer Erfahrung und Untersuchung in seinem Lehrbuch „,̈ber die Ursachen der Lungenkrankheiten" niedergelegt hat.

Tende l o o führt aus, dass exspiratorische Druckerhöhung bei Verengerung bezw. Verschluss der Atemöffnungen zu einer Aufblähung der oberen Lungenpartien führe. Der häufigen akuten Blähung folge die dauernde Überdehnung. Die dauernde Blähung fixiert auch den Thorax in inspiratorischer Stellung und bedingt. die Dehnungsatrophie der Alveolarsepten.

Widerspricht nun diese Ansicht Tendeloos den Ergebnissen unserer spirometrischen Untersuchungen, die in Kernpunkt das gleiche ergaben wie die Untersuchungen Lommels?

Ich glaube diese Frage verneinen zu müssen. Wir haben bei unseren Fällen diese dauernde Blähung eines umschriebenen Lungenabschnittes spirometrisch durch Erhöhung der Lungenmittellage und Residualluft nachweisen können.

Die Feststellung des Sitzes dieser umschriebenen Blähung entzieht sich allerdings auch der spirometrischen Untersuchung vollkommen, ebenso wie die klinische Untersuchung an Lungen und Herz keinerlei Abnormitäten erkennen liess.

Es scheint demnach, - und das sei als Ergebnis unserer Untersuchungen hier ausgesprochen - dass die Ansichten Tendel o o s sehr wohl in Einklang zu bringen sind mit dem Resultate unserer Cntersuchungen. Was der pathologische Anatom, ,als 
Blähung der suprathorakalen Lungenteile, als partielle Überdehnung“ bezeichnet, macht sich spirometrisch in der erhöhten Mittellage und Vermehrung der Residualluft kenntlich.

Wenn wir also hier mit Tendeloo übereinstimmen, so muss andererseits hervorgehoben werden, dass das diffuse chronische Lungenemphysem erfahrungsgemäss meist infolge der durch chronischentzündliche Bronchialverengerung bedingten Atemstörungen eintritt. 


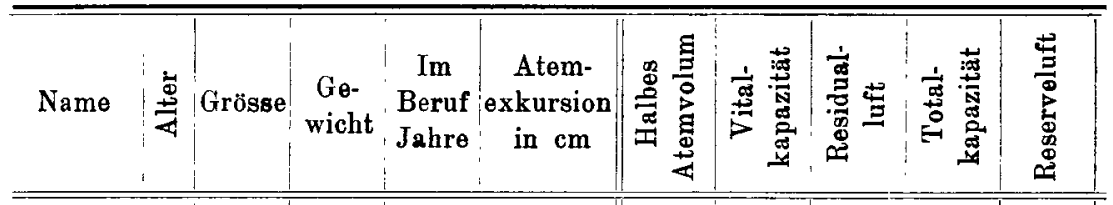

\begin{tabular}{|c|c|c|c|c|c|c|c|c|c|c|}
\hline 1. $\mathrm{K}$. & 20 & 1,68 & 59,5 & 7 & $82: 88$ & 410 & 4500 & 2440 & 6940 & 1500 \\
\hline 2. $\mathrm{Pr}$. & 25 & 1,70 & 70,0 & 11 & $86: 91$ & 550 & 4490 & 2310 & 6800 & 2300 \\
\hline 3. Schm. & 20 & 1,80 & 78,1 & 6 & $88: 96$ & 420 & 5810 & 2470 & 8280 & 2620 \\
\hline 4. Er. & 27 & 1,62 & 61,9 & $13^{1}$ & $87: 91$ & 680 & 5810 & 1070 & 6880 & 2620 \\
\hline 5. R. & 18 & 1,70 & 56,0 & $4^{1 / 2}$ & $83: 88$ & 420 & 3590 & 720 & 4310 & 1700 \\
\hline 6. Sp. & 30 & 1,71 & 70,0 & 14 & $86: 93$ & 440 & 5000 & 1330 & 6330 & 1460 \\
\hline 7. H. & 21 & 1,68 & 67,0 & 8 & $81: 87$ & 320 & 4750 & 1250 & 6000 & 1770 \\
\hline 8. En. & 19 & 1,67 & 59,1 & 5 & $78: 84$ & 280 & 3660 & 1180 & 4840 & 1370 \\
\hline 9. St. & 21 & 1,71 & 66,1 & 7 & $86: 91$ & 270 & 4780 & 1160 & 5940 & 2050 \\
\hline 10. Kr. & 30 & 1,63 & 65,1 & 16 & $88: 3,5$ & 285 & 4130 & 840 & 4970 & 1540 \\
\hline 11. J. & 29 & 1,69 & 79,0 & 16 & $88: 94$ & 340 & 3810 & 1540 & 5350 & 1040 \\
\hline 12. $\mathrm{Bi}$. & 24 & 1,76 & 90,0 & 12 & $97: 101$ & 470 & 4490 & 1320 & 5810 & 1360 \\
\hline 13. Le. & 29 & 1,75 & 81,6 & 16 & $89: 93$ & 500 & 5260 & 1410 & 6670 & 2040 \\
\hline 14. Bl. & 27 & 1,70 & 73,0 & 14 & $92: 96$ & 340 & 4190 & 790 & 4980 & 1340 \\
\hline 15. Bö. & 27 & 1,72 & 69,2 & $121 / 2$ & $84: 92$ & 250 & 4240 & 1260 & 5500 & 1480 \\
\hline 16. L. & 21 & 1,62 & 67,0 & 7 & $85,5: 89,5$ & 285 & 3750 & 1510 & 5260 & 1330 \\
\hline 17. Bre. & 24 & 1,78 & 65,0 & 8 & $82: 89$ & 380 & 4530 & 1220 & 5750 & 1720 \\
\hline 18. Brü. & 25 & 1,69 & 69,0 & 12 & $102: 106$ & $590^{\circ}$ & 4940 & 1670 & 6510 & 1320 \\
\hline 19. M. & 27 & 1,71 & 62,9 & $14^{1 / 2}$ & $78: 84$ & 285 & 4070 & 1450 & 5522 & 1720 \\
\hline 20. V. & 28 & 1,73 & 80,0 & $13^{1} / 2$ & $101: 106$ & 275 & 5230 & 1010 & 6240 & 1780 \\
\hline 21. R. & 26 & 1,72 & 72,0 & 8 & $86: 92$ & 290 & 3710 & 990 & 4700 & 1690 \\
\hline 22. P. & 24 & 1,69 & 67,0 & 10 & $84: 90$ & 305 & 5400 & 1480 & 6880 & 1730 \\
\hline
\end{tabular}




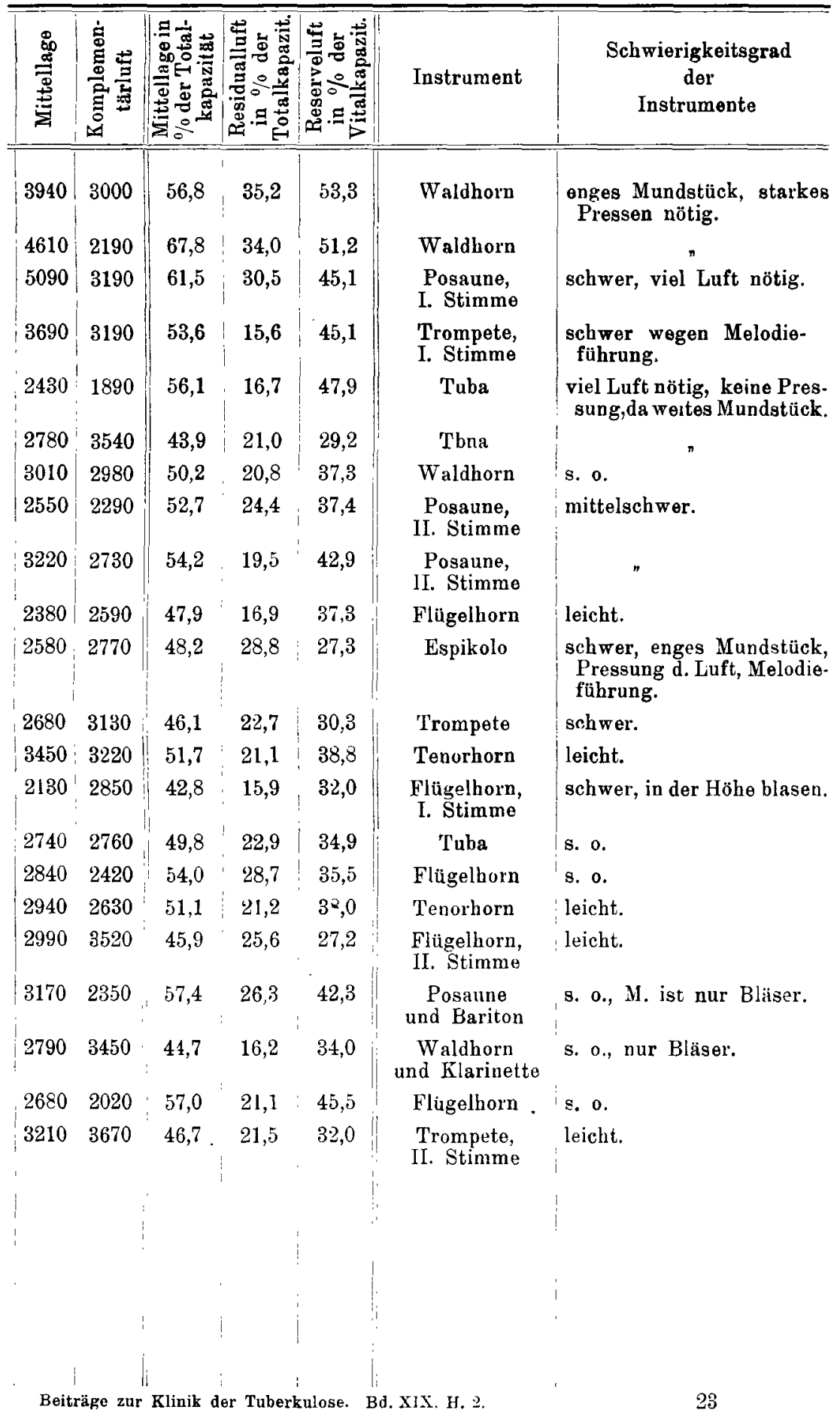


An dieser Stelle möchte ich Gelegenheit nehmen, meinem hochverehrten Lehrer und Chef Herrn Professor Dr. Brauer für die Überlassung der Apparate der Klinik, sowie Herrn Oberarzt Dr. O. Bruns für die Anregung und die dauernde Förderung meiner Arbeit meinen rerbindlichsten Dank auszusprechen.

\section{Literatur.}

1. Bohr, Die funktionelle Änderung in der Mittellage und Vitalkapazität. Deutsch. Arch. f. klin. Med. Bd. 88. S. 385.

2. Bruns, O., Die Bedeutung der spirometrischen Untersuchung von Emphysematikern und Herzkranken. Med. Klinik, 1910. Nr. 39.

3. F is cher, Ist Lungenemphysem eine Eolge des Spielens von Blasinstrumenten? Münch. med. Wochenschr. 1902. Nr. 17.

4. G ebhardt, Über Spirometrie. Münch. med. Wochenschr. 1902. Nr. 47.

5. Hasselbalch, Über die Elinwirkung der Temperatur auf die vitale Mittellage der Lungen.

6. Derselbe, Uber die Totalkapazität der Lungen. Deutsch. Arch. f. klin. Med. Bd. 93. Heft 1 u. 2.

7. Hofmann, Emphysem und Atelektase. Nothnagels Handbuch XIV. 2.

8. Lommel, Zur Pathogenese des Lungenemphysems. Verhandl. d. deutsch. Kongr. f. inn. Med. 27. Kongr. S. 777.

9. Protin und Leibkind, Kann durch Glasblasen ein Lungenemphysem erzeugt werden? Münch. med. Wochenschr. 1904. Heft 6.

10. Strauch, Über die vitale Mittellage der Lungen bei Tuberkulose und Emphysem. Inaug.-Diss. Halle 1910.

11. Tendeloo, Ursachen der Lungenkrankheiten. Wiesbaden, Bergmann 1902.

Bei der Ausrechnung der Tabelle wurde benutzt:

Kohlrausch, Leitfaden der praktischen Physik.

Schlömileh, Fünfstellige Logarithmen. 